\title{
¿Existe un espacio para los análogos de la incretina como terapia para el sobrepeso, la obesidad y la prevención de la enfermedad cardio-metabólica?
}

\author{
José López-López ${ }^{\mathrm{a}}$, Karina A. Di Stefano ${ }^{\mathrm{a}, \mathrm{b}}$, Elizabeth Velásquez ${ }^{\mathrm{a}}$, \\ Paul Anthony Camacho ${ }^{\mathrm{a}, \mathrm{c}}$ y Patricio López-Jaramillo ${ }^{\mathrm{a}, \mathrm{b}, *}$
}

\begin{abstract}
a Dirección de Investigaciones, Desarrollo e Innovación Tecnológica, Fundación Oftalmológica de Santander (FOSCAL), Floridablanca, Colombia

b Instituto MASIRA, Facultad de Medicina, Universidad de Santander (UDES), Bucaramanga, Colombia

c Facultad de Ciencias de la Salud, Universidad Autónoma de Bucaramanga (UNAB), Bucaramanga, Colombia
\end{abstract}

Recibido el 1 de julio de 2015; aceptado el 13 de octubre de 2015

Disponible en Internet el 29 de noviembre de 2015

\section{PALABRAS CLAVE \\ Obesidad; \\ Factores de riesgo \\ cardiovascular; \\ Enfermedad \\ cardiovascular}

\begin{abstract}
Resumen
Introducción: En las últimas décadas se ha producido un aumento progresivo de la enfermedad cardiovascular, convirtiéndose en la principal causa de mortalidad en el mundo y especialmente en los países de Latinoamérica, a pesar de haberse identificado los factores de riesgo cardiovascular prevenibles y que explican alrededor del $90 \%$ de la causalidad de un infarto de miocardio y de un accidente cerebrovascular. Por tanto, es necesario implementar las acciones que permitan controlar los factores de riesgo cardiovascular incluyendo sobrepeso y obesidad. Objetivo: Revisar el efecto que tienen los análogos de los receptores del GLP-1, entre ellos la liraglutida, en el control de factores de riesgo cardiovascular, particularmente del sobrepeso y la obesidad y en la consecuente prevención de la enfermedad cardiovascular.

Métodos: Se realizó una búsqueda de la evidencia con operadores booleanos y referencias cruzadas en base de datos MEDLINE, LILACS y Google Scholar.

Conclusiones: Los análogos de los receptores de GLP-1 pueden ser una alternativa farmacológica para el manejo del sobrepeso, la obesidad y contribuir en la prevención de la enfermedad cardiovascular, aunque todavía quedan interrogantes sobre el beneficio de su uso en nuestro medio.

(C) 2015 Sociedad Colombiana de Cardiología y Cirugía Cardiovascular. Publicado por Elsevier España, S.L.U. Este es un artículo Open Access bajo la CC BY-NC-ND licencia (http:// creativecommons.org/licencias/by-nc-nd/4.0/).
\end{abstract}

\footnotetext{
* Autor para correspondencia.

Correo electrónico: jplopezj@gmail.com (P. López-Jaramillo).
} 


\section{KEYWORDS}

Obesity;

Cardiovascular risk

factors;

Cardiovascular

disease

\section{Is there a space for incretin mimetics as a therapy for excess weight, obesity and cardiometabolic disease prevention?}

\begin{abstract}
Introduction: During the last decades there has been a gradual increase of cardiovascular diseases, who have become the main cause of death in the world and especially in Latin American countries, despite having identified preventable cardiovascular risk factors that explain around $90 \%$ of myocardial infarctions and cerebrovascular accidents. Therefore, it is require to implement actions that will allow to control cardiovascular risk factors, including being overweight or obese.

Motivation: To review the effect of GLP-1 receptor agonists, including liraglutide, in controlling cardiovascular risk factors, particularly excess weight and obesity and the subsequent prevention of cardiovascular diseases.

Methods: Evidence were searched by means of boolean operators and cross references in databases MEDLINE, LILACS and Google Scholar.

Conclusions: GLP-1 receptor agonists can be a pharmacological alternative to control excess weight and obesity, and may help in preventing cardiovascular disease, though some questions remain regarding the benefits of its use in our field.

(c) 2015 Sociedad Colombiana de Cardiología y Cirugía Cardiovascular. Published by Elsevier España, S.L.U. This is an open access article under the CC BY-NC-ND license (http:// creativecommons.org/licenses/by-nc-nd/4.0/).
\end{abstract}

\section{Introducción}

La enfermedad cardiovascular es la primera causa de mortalidad en el mundo, explicando alrededor del $30 \%$ de todas las muertes. En países con altos ingresos se ha logrado un descenso de la mortalidad por causas cardiovasculares, mientras que en países con medianos y bajos ingresos existe una tendencia opuesta. Las proyecciones de la Organización Mundial de la Salud (OMS) indican que en las próximas tres décadas, la mortalidad cardiovascular se doblará a nivel global, fenómeno atribuible al aumento de la mortalidad cardiovascular de estos países. De hecho, el aumento en la mortalidad por la enfermedad cardiaca isquémica prevista para Latinoamérica en las próximas tres décadas alcanza el $140 \%{ }^{1}$.

La identificación de los factores de riesgo cardiovascular (FRCV) que hoy se conocen como convencionales han permitido predecir una proporción creciente del riesgo de un evento cardiovascular (v.g. infarto agudo de miocardio o enfermedad cerebrovascular aguda). El estudio INTERHEART (que incluyó aproximadamente 21.000 casos con infarto agudo de miocardio y similar número de controles pareados en 52 países, con participantes de Colombia) estimó que nueve factores de riesgo prevenibles explican alrededor del $90 \%$ del riesgo atribuible a la población para la ocurrencia de un primer infarto agudo de miocardio ${ }^{2}$. En América Latina la mayoría de la causalidad atribuible es dada, además, del tabaquismo, por: obesidad abdominal, dislipidemia, hipertensión arterial y Diabetes Mellitus tipo 2 (DM2), conjunto de alteraciones que conforman el denominado Síndrome Metabólico y que en Latinoamérica es de particular importancia por su alta prevalencia que llega a ser de más del $35 \%^{3}$. El estudio INTERSTROKE ${ }^{4}$ reportó que este conjunto de factores de riesgo fueron similares para el accidente cerebrovascular isquémico o hemorrágico, y además, que en los pacientes sudamericanos la obesidad abdominal, junto con la hipertensión y el tabaquismo fueron los FRCV más comunes. Estos datos son particularmente relevantes para nuestra región dado el dramático incremento del sobrepeso y la obesidad observado en las últimas décadas que determinan que uno de cada dos adultos estén en sobrepeso $\mathrm{u}$ obesidad $^{5}$, y es bien conocido que el exceso de grasa corporal aumenta el riesgo de DM2 y ECV por inducir, entre otros mecanismos, un estado de resistencia a la insulina, de inflamación sistémica de bajo grado y por alterar la reactividad vascular $^{6-9}$.

Actualmente, se estima que alrededor de 250 millones de personas viven con DM2 y se espera que este número se incremente aproximadamente a 380 millones en el año 2025. La Federación Internacional de Diabetes planteó que 5.1 millones de personas entre 20 y 79 años de edad fallecieron por DM2 en el año $2013^{10}$. Esta cifra puede ser mucho mayor si se considera que un alto número de fallecimientos fueron registrados como muerte cardiaca o falla renal, situaciones en las cuales la DM2 contribuye de forma sustancial para el desenlace fatal de estas enfermedades. Desde el punto de vista económico, el tratamiento de la DM2 y de sus complicaciones en el mundo entero cuesta alrededor de 232 billones de dólares (estimaciones para el año 2007), y se estima que para el año 2025 subirá a más de 302 billones de dólares ${ }^{11,12}$. Por tanto, la DM2 no es solo un serio problema de salud pública, sino también un enorme problema económico que puede ser especialmente grave para países de economías débiles como los latinoamericanos. La carga económica que estas cifras representarán para el sistema de salud de estos países será enorme, en consideración de las mayores tasas de hospitalización y de cuidados médicos que tienen los pacientes con DM2, por la mayor incidencia de: la enfermedad cardiovascular, la ceguera, la insuficiencia renal y las amputaciones no traumáticas de miembros inferiores ${ }^{13}$. En Colombia, la Encuesta Nacional de Salud y Nutrición (ENSIN) en el año $2007^{14}$, demostró 
que la prevalencia de DM2 fue de $5 \%$ (glicemia capilar en ayunas igual o mayor a $126 \mathrm{mg} / \mathrm{dl}$ ) y la de prediabetes $9 \%$ (glicemia capilar en ayunas de $100 \mathrm{a} 125 \mathrm{mg} / \mathrm{dl}$ ).

Estos datos determinan la urgente necesidad de establecer acciones de salud pública dirigidas a implementar cambios en los hábitos de vida y a establecer la utilidad de intervenciones con medicamentos que permitan controlar: la epidemia de sobrepeso, la obesidad y la diabetes, basados en los recientes conocimientos sobre la fisiopatología de la resistencia a la insulina y la DM2 entre los cuales se destaca el eje incretínico, en el cual se profundizará en esta revisión.

\section{Métodos}

Se realizó una búsqueda de la evidencia en Medline, LILACS y Google Académico. Se utilizaron los términos MESH, DECs y texto libre «liraglutida», «análogos del receptor del GLP-1», «diabetes mellitus 2», «obesidad», «obesidad abdominal», «síndrome metabólico», «factores de riesgo cardiovascular», «enfermedad cardiovascular» en inglés y español. La construcción de la búsqueda se hizo con operadores booleanos y referencias cruzadas de los artículos identificados. Los criterios de elegibilidad para la selección de los artículos fueron: ensayos clínicos aleatorizados, en inglés y español, sobre la liraglutida comparado con otras estrategias terapéuticas (placebo, dieta, sulfonilureas, tiazolidindionas, insulina $u$ otros), los pacientes con diabetes mellitus tipo 2, con una intervención mínima de 8 semanas y reporte sobre el peso. Dos revisores (JLL y KDD), independientemente, revisaron y evaluaron títulos y resúmenes identificados en la búsqueda. Se eliminaron duplicaciones. Se extrajeron los datos que nos permitieron explicar el efecto que tienen los análogos de los receptores del GLP1 , entre ellos la liraglutida, en el control de factores de riesgo cardiovascular y en la prevención de la enfermedad cardiovascular.

\section{Resultados}

En la búsqueda se identificaron 121 artículos, de éstos fueron excluidos 115 por no cumplir los criterios de elegibilidad. Se seleccionaron 4 ensayos clínicos aleatorizados, con 7.324 participantes con la liraglutida y el placebo, que cumplieron los criterios de inclusión (fig. 1).

\section{El eje entero-pancreático}

Alrededor del año 1920 se introdujo el término incretina que fue el primero en ser usado para definir los factores que regulan la secreción del páncreas, no obstante, la conexión entre el tracto gastrointestinal y el páncreas solo se propusó a finales de la década de 1960 cuando la insulina se empezó a medir en el plasma. Los estudios clínicos de la época demostraron una mayor respuesta secretora de insulina con la administración de la glucosa oral en comparación a la intravenosa a pesar de producir los mismos aumentos de glucemia. Estos hallazgos sugirieron que el incremento de la glucemia, luego de una ingesta oral de glucosa, interactuaba con las células beta en los islotes de Langerhans, pero que también otros factores eran liberados en el tracto

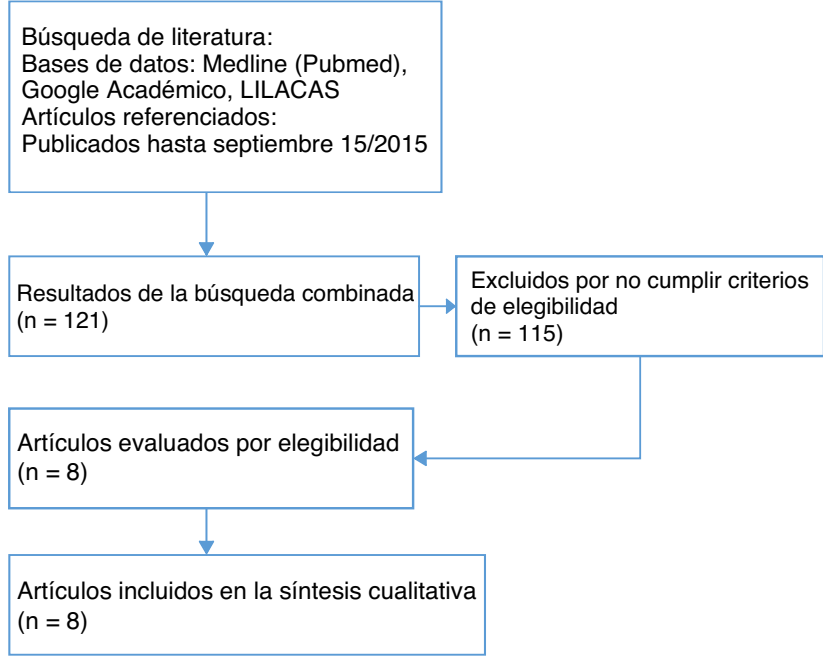

Figura 1 Flujograma de búsqueda: resultados de la búsqueda, artículos potencialmente elegibles, incluidos y excluidos.

gastrointestinal y que estos estimulaban la secreción de insulina $^{15}$. Fue así como en el año 1979 e inicios del año 1980 se describió el eje entero pancreático, el cual utiliza dos hormonas principales: el Péptido 1 semejante al Glucagón (GLP-1 por sus siglas en inglés; Glucagon-Like Peptide 1) y el Péptido Insulinotrópico dependiente de Glucosa (GIP; Glucose-dependent Insulinotropic Peptide). Estas hormonas insulinotrópicas se secretan en respuesta a la ingestión oral de la glucosa y permiten mantener los niveles apropiados de la insulina plasmática y de la glicemia en respuesta a la ingesta de alimentos. Se ha estimado que del 50 al $70 \%$ de la respuesta de la insulina a la ingesta de la glucosa oral es atribuible al sistema incretínico ${ }^{16}$.

Alteraciones en el eje incretínico tienen un importante papel en el desarrollo de la disfunción de la célula beta pancreática ${ }^{17}$, y en el consecuente desarrollo de DM2 y se ha descrito que los sujetos con DM2 tienen el efecto incretínico disminuido ${ }^{18}$. Estas observaciones llevaron a estudiar la posibilidad de utilizar el GLP-1 como terapia para el control de la $\mathrm{DM} 2^{19}$, consecuentemente para prevenir el progreso de la enfermedad y la aparición de las complicaciones vasculares.

El GLP-1 es un péptido de 30 aminoácidos que se sintetiza a partir de la ruptura del producto de transcripción del gen que regula la síntesis del preproglucagón ${ }^{20}$. La mayoría del GLP-1 es producido por la células entero-endocrinas $L$ en el íleon distal y el colón, alcanzando sus niveles plasmáticos más altos en los primeros minutos después de la alimentación (fig. 2). La vida media biológica del GLP-1 in vivo es corta (aproximadamente 2 minutos) y se degrada rápidamente por la acción de la enzima dipeptidil dipeptidasa-4 (DPP-4) $)^{21}$, por tanto, el enfoque farmacológico ha sido dirigido a prolongar la vida media del GLP-1, ya sea por modificación estructural del péptido original o por inhibición de la enzima DDP-4. Existen diferentes agonistas del receptor GLP-1 aprobados para el manejo de la DM2 así: el exenatide (comercializado como Byetta ${ }^{\circledR}$; Eli Lilly \& Co, Indianapolis, IN, USA) con aplicación dos veces al día, la liraglutida (comercializado como Victoza ${ }^{\circledR}$; Novo Nordisk, Copenhague, Dinamarca) y el lixisenatide (comercializado como Lyxumia $^{\circledR}$, Sanofi, París, Francia) con aplicación una vez al 


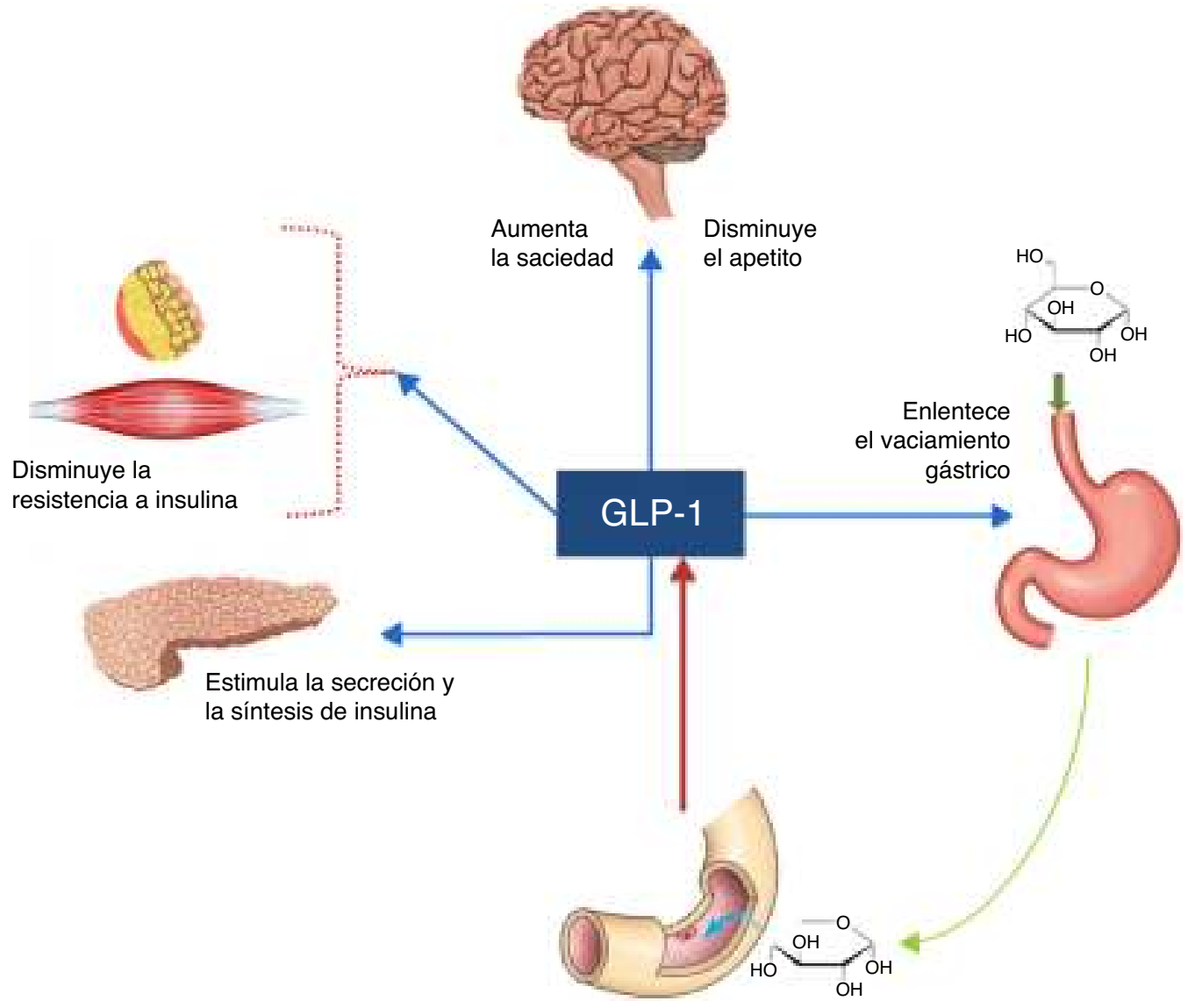

Figura 2 Eje entero-pancreático.

día. Actualmente están también en el mercado: el exenatide de liberación prolongada y el albiglutide (Tanzeum $^{\circledR}$; GlaxoSmithKline, Londres, Inglaterra) que son agonistas de acción prolongada y que se aplican semanalmente.

Se revisarán a continuación las características de la liraglutida, el incretino-mimético que ha sido aprobado por la Oficina Americana de Administración de Drogas y Alimentos (FDA), como tratamiento adyuvante para la obesidad. La liraglutida es un fármaco análogo del receptor de GLP-1 que se obtiene de la sustitución de Lys por Arg en la posición 34 y la adición de un ácido graso de 16 cadenas (ácido palmitoil) a Lys en la posición 26 del GLP-1. Estas modificaciones mantienen una secuencia de aminoácidos que es similar en un $97 \%$ con respecto a la secuencia original del GLP-1 corporal $^{22}$. La liraglutida se administra por vía subcutánea mediante un lapicero multidosis. Su tiempo de acción es de 9 a 13 horas, motivo por el que se puede administrar una dosis diaria. La prolongación de su acción se debe a la agregación en el sitio de inyección, a la unión a la albúmina en la circulación y a la disminución en la susceptibilidad a la degradación por parte de la enzima DDP-422-24. Datos in vitro sugieren que la liraglutida es degradada de manera similar al GLP-1 corporal por endopeptidasas neutras, así como por DDP $-4^{25}$. La degradación del fármaco no se altera cuando hay disminución de la función renal o hepática ${ }^{26}$. En evaluaciones in vitro la liraglutida tiene bajo potencial de interacción medicamentosa con productos relacionados con citocromo P450 y con aquellos que se unen a las proteínas plasmáticas $^{27}$.
La administración de la liraglutida tiene efectos agudos similares a GLP-1 en el censo de la glucosa y la secreción de insulina a través del cierre de los canales de $\mathrm{K}$ dependientes de ATP, cambio en el potencial de la membrana y sensibilización de la célula beta pancreática a la glucosa ${ }^{28}$, liberación de los depósitos internos de calcio iónico ${ }^{29}$, y disminución de los canales de $\mathrm{K}$ dependientes de voltaje $\mathrm{e}^{30}$. Cuando es administrada crónicamente el efecto incretínico se caracteriza por aumento de los niveles de RNAm de insulina ${ }^{31}$ y de los niveles de los factores de transcripción de RNAm de insulina. Por tanto, la liraglutida tiene un efecto inmediato estimulando la secreción de insulina y un efecto a largo plazo en la estimulación de síntesis de insulina. A nivel de sistema nervioso central, el GLP-1 es producido por neuronas que inervan las áreas mesolímbicas tales como: el área tegmental ventral (ATV) y el núcleo acumbens (NA). El ATV modula el comportamiento de recompensa ${ }^{32}$, y sus proyecciones dopaminérgicas al NA orquestan una conducta motivada para obtener los reforzamientos naturales tales como: la comida y el sexo $0^{33,34}$. El GLP-1 es producido en el núcleo del tracto solitario ${ }^{35}$, el cual recibe las aferencias vagales del tracto gastrointestinal y las aferencias gustatorias de la lengua ${ }^{36}$, por lo que se convierte en una localización estratégica para que las neuronas productoras del GLP-1 puedan tener un control sobre el balance de energía. El GLP-1 actuando en sus receptores ubicados en estas áreas ejerce un efecto anorexigénico directo en el hipotálamo y los núcleos del tronco encefálico ${ }^{37,38}$, mediante la reducción del sistema de recompensa. Desde el punto de 
vista clínico, esto tiene importancia debido a que la disminución en la ingesta coadyuva a la pérdida de peso en los pacientes con obesidad. Además, actuando en el estómago e intestino delgado donde existen receptores del GLP-1, produce un retraso del vaciamiento gástrico, acción que ha sido propuesta como beneficiosa en perspectiva de explicar la pérdida de peso que se observa en algunos pacientes bajo tratamiento con la liraglutida ${ }^{39,40}$, pero también, la mayoría de efectos adversos como: las náuseas y los vómitos se relacionan con esta acción, la cual determina que aproximadamente el $3 \%$ de los pacientes descontinúen el tratamiento ${ }^{41}$. Estos efectos adversos, en general, son leves y transitorios. Debido a que la acción de la liraglutida es dependiente de la glucosa, el riesgo de hipoglicemia severa es bajo como se ha observado en los estudios fase $3^{42}$.

\section{Mecanismos de acción alternativos}

Entre los mecanismos propuestos para explicar la pérdida de peso ocasionada por la liraglutida se ha propuesto un efecto central inhibiendo el apetito ${ }^{43}$. Esta propuesta se basa en trabajos experimentales en un modelo de ratas prediabéticas en los que se demostró un efecto agudo de la liraglutida en la disminución de la ingesta de alimentos, sin embargo, posteriormente el efecto en la pérdida de peso se mantuvo independiente de la ingesta de comida, lo que sugiere que el efecto de la liraglutida sobre la pérdida de peso puede ejercerse a través de otros mecanismos adicionales diferentes a la sola ingesta de alimentos ${ }^{44}$. Cummings, et al. ${ }^{45}$ en un modelo poligénico de ratas con obesidad y DM2 reportaron que los animales en tratamiento con la liraglutida presentaron una reducción sostenida de la ingesta de alimentos y un menor porcentaje de masa grasa corporal en relación con animales en control mantenidos con alimentación restringida que presentaron la misma pérdida de peso, lo que sugiere un efecto del incretino mimético en la distribución de grasa corporal. En soporte de esta propuesta un estudio reciente realizado en 20 sujetos japoneses con DM2 hospitalizados, en los cuales después de lograr el control glicémico con insulina se cambió el tratamiento hipoglucemiante a la liraglutida, demostró en el análisis de impedancia bioeléctrica abdominal disminuciones significativas en el perímetro abdominal $(4,28 \pm 0,55 \mathrm{~cm})$, del índice cintura/cadera y en el área estimada de grasa visceral (AEGV), después de $20 \pm 6,4$ semanas de tratamiento con la liraglutida en rango de 0,3 a 0,9 mg/ día ${ }^{46}$, datos confirmados por el estudio LEAD-2 que demostró una reducción significativa del perímetro abdominal y del AEGV en los pacientes diabéticos que recibieron la liraglutida comparados con los que se trataron con la glimepirida ${ }^{47}$.

Además, de los efectos positivos de los análogos del GLP-1 en la pérdida de peso y en los parámetros metabólicos de los pacientes con sobrepeso y obesos sin DM2 y en aquellos obesos prediabéticos y diabéticos ${ }^{48}$, la liraglutida tiene similar efecto que la incretina endógena en la preservación de la función de la célula beta ${ }^{49}$. En los estudios LEAD 1, 2 y 5 (Liraglutide Effect and Action in Diabetes) se midió el índice HOMA como indicador de función de la célula beta, observándose mejorías de 28 -34\% luego del tratamiento con la liraglutida ${ }^{50,51}$, y los estudios SCALE ${ }^{52-54}$, demostraron que disminuye la progresión de la obesidad a prediabetes en los obesos normo glicémicos que recibieron $3 \mathrm{mg} /$ día de la liraglutida, grupo en el cual solo el $6.9 \%$ presentaron valores glicémicos de prediabetes a las 56 semanas mientras que $19.9 \%$ de los sujetos que recibieron placebo desarrollaron prediabetes. En las ratas obesas prediabéticas OLETF (Otsuka-Long-Evans-Tokushima Fatty rats) se observó que después de 12 semanas de tratamiento con la liraglutida existía una adecuada conservación de la morfología de los islotes pancreáticos, regulación a la alta de la expresión de factor antiapoptótico Bcl-2 y regulación a la baja de la expresión del factor proapoptótico Bax, resultados que sugieren que la liraglutida tiene un papel protector en la formación y la función del islote pancreático mediante el control de las vías apoptóticas, lo que permite mantener la función endocrina de la célula beta y prevenir las alteraciones de la glucosa y la aparición de $\mathrm{DM} 2^{44}$.

\section{Control del sobrepeso, la obesidad y la diabetes mellitus tipo 2}

Los estudios LEAD fueron diseñados para investigar los efectos de la liraglutida en los pacientes con DM2 de acuerdo a la secuencia de escalonamiento del tratamiento de la DM2 dadas por las guías terapéuticas: iniciando como terapia única después del fracaso en la implementación de cambios terapéuticos en los hábitos de vida y finalizando como parte de terapia múltiple combinada con uno o dos antidiabéticos orales (ADO).

El estudio LEAD $3^{55}$, que evaluó la liraglutida como monoterapia en 746 sujetos que estaban mal controlados con dieta y ejercicio y con no más de la mitad de la dosis máxima de un $\mathrm{ADO}$, demostró una disminución significativa de $\mathrm{HbA} 1 \mathrm{c}$ con la liraglutida 1,8 mg/día $(1,1 \%, p<0,0001)$ o 1,2 mg/día $(0,8 \%, p=0,0014)$ en comparación con $8 \mathrm{mg}$ de la glimepirida $(0,5 \%)$. Cuando se evaluó el efecto de la liraglutida en combinación con un ADO en el estudio LEAD 2 se observó que la combinación de $1,8 \mathrm{mg}$ de la liraglutida más la metformina disminuye en $1 \%$ la $\mathrm{HbA} 1 \mathrm{c}$ en comparación con la metformina sola y hubo similar control glicémico, mayor pérdida de peso y menor ocurrencia de hipoglicemia en comparación con la glimepirida ${ }^{56}$. El estudio LEAD 1 demostró que la terapia combinada con $1,8 \mathrm{mg}$ de liraglutida más $4 \mathrm{mg}$ de glimepirida produjo mayor reducción en los niveles de $\mathrm{HbA1c}$ basales $(-1,1 \%$, basales $8,5 \%)$ comparado con placebo $(+0,2 \%$, $p<0,0001$, basales $8,4 \%$ ) o con la combinación de la rosiglitazona más la glimepirida $(-0,4 \%, p<0,0001 \text {, basales } 8,4 \%)^{42}$. Generalmente, el tratamiento con la liraglutida produce una disminución de 0,8 a $1,5 \%$ en los niveles de $\mathrm{HbA} 1 \mathrm{c}$, asociado a la pérdida de peso y la disminución en la frecuencia de hipoglicemias ${ }^{56}$. Este conjunto de resultados beneficiosos para la mayoría de los pacientes diabéticos demuestran que los análogos del GLP-1 deben ser considerados en el momento de establecer un tratamiento óptimo para la DM2, aunque todavía no se ha demostrado consistentemente, que estos fármacos son efectivos también en la disminución de las complicaciones vasculares asociadas a la obesidad, la prediabetes y la $\mathrm{DM} 2^{57}$. Al momento existen algunas evidencias indirectas que sugieren que los agonistas del receptor del GLP-1 tienen el potencial de reducir el riesgo de la enfermedad cardiovascular en pacientes con $\mathrm{DM2}^{54}$. Así, la disminución de la presión arterial, así sea mínima, ha 
mostrado disminuir el riesgo de enfermedad cardiovascular y en los estudios LEAD se encontró una disminución de la presión arterial (PA) clínicamente significativa desde $-2,1 \mathrm{mmHg}$ en LEAD 3 a $-6,7 \mathrm{mmHg}$ en LEAD 4 42,55,58,59. No obstante, la hipertensión no fue un desenlace en los estudios LEAD por lo que no se discriminó el uso de medicamentos antihipertensivos concomitantes. Sin embargo, Fonseca, et al. ${ }^{60}$, en un análisis agrupado de seis estudios clínicos en pacientes con DM2 mostró que la liraglutida produjo una reducción significativa de la PA en individuos con o sin utilización de medicamentos antihipertensivos concomitantes. Del mismo modo, Robinson, et al. ${ }^{61}$, en un metaanálisis de 32 estudios clínicos mostró que los agonistas del GLP-1 disminuyen la PAS en $-1,79 \mathrm{mmHg}(-2,94$ a $-0,64)$ y $-2,39 \mathrm{mmHg}(-3,35$ a $-1,42)$, comparado a placebo y control activo, respectivamente. $Y$ se ha propuesto que la reducción de la PA está asociada a la pérdida de peso, aunque la correlación entre reducción de la PA y la pérdida de peso fue muy débil, por lo que se ha sugerido que la disminución de la PA puede deberse a la activación de los receptores del GLP-1 que llevan a un aumento en la natriuresis y la vasodilatación ${ }^{62,63}$. La eventual acción benéfica de la liraglutida en el riesgo cardiovascular en pacientes con DM2 está siendo investigado en el estudio LEADER (Liraglutide Effect and Action in Diabetes: Evaluation of Cardiovascular Outcome Results) ${ }^{64}$.

Un reciente metaanálisis ${ }^{65}$, que incluyó 21 estudios clínicos aleatorizados y 3.395 sujetos asignados a agonistas del GLP-1 (la liraglutida y la exenatida) y 3.016 individuos asignados a control (placebo, antidiabéticos orales, insulina) mostró un efecto beneficioso de los agonistas del receptor GLP-1 en la pérdida de peso en pacientes con sobrepeso y obesidad, con o sin DM2. Tres ensayos clínicos aleatorizados realizados en 594 pacientes sin DM2, demostraron una pérdida de peso promedio de $-3,2 \mathrm{~kg}(-4,3 \mathrm{a}-2,1 \mathrm{~kg})$, mientras que en 18 estudios que incluyeron pacientes con DM2 se observó una pérdida de peso promedio de $2,8 \mathrm{~kg}(-3,4$ a $-2,3 \mathrm{~kg})$. Astrup, et al. ${ }^{66}$, utilizaron la liraglutida para el tratamiento de la obesidad en 564 sujetos con índice de masa corporal entre 30 y $40 \mathrm{~kg} / \mathrm{m}^{2}$, los cuales fueron asignados al azar a tres brazos: la liraglutida (cuatro dosis 1,2, 1,8, 2,8, $3 \mathrm{mg}$ ), el orlistat o el placebo y los tres grupos recibieron una dieta con moderada restricción calórica. El grupo en la liraglutida mostró una mayor pérdida de peso $(4,8-7,2 \mathrm{~kg})$ en comparación al grupo en el orlistat $(4,1 \mathrm{~kg})$ o en el placebo $(2,8 \mathrm{~kg})$, pérdidas de peso que se mantuvieron en los grupos con la liraglutida durante los 2 años de tratamiento ${ }^{67}$. En la tabla 1 se resumen los estudios clínicos aleatorizados que evaluaron la diferencia de peso corporal con la utilización de la liraglutida.

Recientemente, se publicaron ${ }^{52,53}$, los resultados del estudio SCALE (Satiety and Clinical Adiposity-Liraglutide Evidence in Nondiabetic and Diabetic Individuals), ensayo clínico aleatorizado, doble ciego, controlado con placebo que incluyó 3.731 pacientes obesos con más de 30 de IMC o con más de 27 de IMC si tenían dislipidemia o hipertensión tratada o no ${ }^{52}$, y 846 pacientes con DM2 y con IMC igual o mayor a 27 tomando o no agentes hipoglicemiantes y cuyas $\mathrm{HbA} 1 \mathrm{C}$ se encontraban entre 7,0 y $10,0 \%{ }^{53}$. En el primer estudio todos los pacientes en el grupo activo (2.487) recibieron $3,0 \mathrm{mg} /$ día de la liraglutida por 56 semanas y 1.244 recibieron el placebo. En el segundo estudio 423 pacientes con DM2 recibieron $3,0 \mathrm{mg} /$ día de la liraglutida mientras

Tabla 1 Diferencia en el peso corporal de estudios clínicos aleatorizados con utilización de la liraglutida que incluyeron únicamente pacientes sin diabetes

\begin{tabular}{|c|c|c|c|c|}
\hline Estudio & Dosis de la Liraglutida & n pacientes & Peso inicial kg & Diferencia en el peso corporal kg \\
\hline \multirow[t]{5}{*}{ Astrup $2009^{66}$} & $1,2 \mathrm{mg}$ & 95 & $96,2(13,5)$ & $-6,7(4,0)$ \\
\hline & $1,8 \mathrm{mg}$ & 90 & $98,0(12,5)$ & $-7,1(5,8)$ \\
\hline & $2,4 \mathrm{mg}$ & 93 & $98,4(13,0)$ & $-7,9(5,0)$ \\
\hline & $3,0 \mathrm{mg}$ & 93 & $97,6(13,7)$ & $-9,1(5,2)$ \\
\hline & Placebo & 98 & $97,3(10,3)$ & $-4,1(3,9)$ \\
\hline \multirow[t]{2}{*}{ Garber $2009^{55}$} & $1,2 \mathrm{mg}$ & 251 & $92,5(19,2)$ & $-3,24$ \\
\hline & $1,8 \mathrm{mg}$ & 247 & $92,8(20,7)$ & $-3,39$ \\
\hline \multirow[t]{3}{*}{ Marre $2009^{42}$} & $1,2 \mathrm{mg}$ & 228 & $80,0(17,1)$ & $+0,3$ \\
\hline & $1,8 \mathrm{mg}$ & 234 & $83,0(18,1)$ & $-0,2$ \\
\hline & Placebo & 114 & $81,9(18,1)$ & $-0,1$ \\
\hline \multirow[t]{2}{*}{ Russel-Jones $2009^{59}$} & $1,8 \mathrm{mg}$ & 230 & $85,5(19,4)$ & $-1,39(5,4)$ \\
\hline & Placebo & 114 & $85,7(16,7)$ & $-0,42(0,39)^{*}$ \\
\hline \multirow[t]{3}{*}{ Zinman $2009^{58}$} & $1,2 \mathrm{mg}$ & 178 & & $-1,0(0,3)$ \\
\hline & $1,8 \mathrm{mg}$ & 178 & & $-2,0(0,3)$ \\
\hline & Placebo & 177 & & $0,6(0,3)$ \\
\hline \multirow[t]{2}{*}{ Wadden $2013^{54}$} & $3,0 \mathrm{mg}$ & 212 & $106,7(22,0)$ & $-6,0(7,3)$ \\
\hline & Placebo & 210 & $105,0(22,5)$ & $-0,1(6,9)$ \\
\hline \multirow[t]{2}{*}{ Pi-Sunyer $2015^{52}$} & $3,0 \mathrm{mg}$ & 2487 & $106,2(21,4)$ & $-.8,4(7,3)$ \\
\hline & Placebo & 1244 & $106,2(21,7)$ & $-2,8(6,5)$ \\
\hline \multirow[t]{3}{*}{ Davies $2015^{53}$} & $3,0 \mathrm{mg}$ & 423 & $105,7(21,9)$ & $-6,4$ \\
\hline & $1,8 \mathrm{mg}$ & 211 & $105,8(21,0)$ & $-5,0$ \\
\hline & Placebo & 212 & $106,5(21,3)$ & $-2,2$ \\
\hline
\end{tabular}

Promedio (Desviación Estándar).

* Error Estándar. 
211 recibieron $1,8 \mathrm{mg} /$ día y 212 recibieron el placebo. Los pacientes con sobrepeso y obesos sin DM tuvieron un peso inicial promedio de $106,2 \pm 21,4 \mathrm{~kg}$ y un IMC promedio de $38,3 \pm 6,4$. Los pacientes asignados a la liraglutida tuvieron a la semana 56 del estudio una pérdida de peso promedio de $8,4 \pm 7,3 \mathrm{~kg}$, mientras que los del grupo del placebo tuvieron una pérdida promedio de $2,8 \pm 6,5 \mathrm{~kg}$. Un total de $63,2 \%$ en el grupo de la liraglutida perdieron más de $5 \%$ de peso, mientras que en el grupo del placebo lo hizo solo el $27,1 \%$. En el grupo activo $33,1 \%$ perdió más del $10 \%$ de peso mientras en el grupo placebo este porcentaje llego solo a $10,6 \%$. Estos resultados en una muestra más amplia de pacientes con sobrepeso y obesos sin DM2 confirmaron los hallazgos observados en una muestra más reducida ${ }^{54}$. Los pacientes con DM2 del segundo estudio tuvieron un peso promedio inicial de $105,7,105,8$ y 106,5 kg en cada uno de los grupos. Al final del estudio los pacientes del grupo de $3,0 \mathrm{mg} /$ día de la liraglutida perdieron en promedio $6,4 \mathrm{~kg}(6,0 \%)$, los de 1,8 perdieron en promedio $5,0 \mathrm{~kg}(4,7 \%)$, mientras los del grupo placebo perdieron $2,2 \mathrm{~kg}(2,0 \%)$. Mientras en el grupo con la dosis alta de la liraglutida $54,3 \%$ perdieron más del $5 \%$ de su peso inicial, $40,4 \%$ lo hicieron con la dosis menor y solo $21,4 \%$ en el grupo placebo.

En las conclusiones de los autores del SCALE se destaca que la administración de $3 \mathrm{mg} /$ día de la liraglutida como un tratamiento adicional a la dieta y el ejercicio, se asocia con un pérdida de peso clínicamente importante en pacientes con sobrepeso y obesos diabéticos o no, con reducciones concomitantes en la concentración de glucosa y de otros factores de riesgo cardiovascular y con una mejoría en la calidad de vida.

\section{Resultados de seguridad}

En las recomendaciones de seguridad para el uso de la liraglutida de la Agencia Americana de Alimentos y Medicamentos (FDA, Food and Drug Administration), se señala el posible aumento en el riesgo de desarrollar pancreatitis debido al mecanismo de acción de los GLP-1 ${ }^{68,69}$. Esta preocupación surgió por el reporte de varios casos de pancreatitis debido al uso del exenatide y la sitagliptina, moléculas con mecanismos de acción similares a los de la liraglutida. Sin embargo, dos estudios aleatorizados, doble ciego, que incluyeron 16.492 pacientes y 5.380 pacientes con DM2, el Saxagliptin Assessment of Vascular Outcomes Reported $(\text { SAVOR })^{70}$ y el Examitation of Cardiovascular Outcomes with Alogliptin versus standar of care $\left(\right.$ EXAMINE) ${ }^{71}$, reportaron tasas bajas de pancreatitis aguda similares a los grupos placebo (EXAMINE, 22 y 16 pacientes) y (SAVOR, 12 y 8 pacientes). Un metaanálisis ${ }^{72}$, que incluyó 25 estudios clínicos aleatorizados para evaluar la presencia de eventos adversos serios como pancreatitis aguda y cáncer con el uso de la liraglutida y el exenatide, documentó no asociación de estos fármacos con dichos eventos, y se concluyó que no hay evidencia suficiente para considerar que estos eventos sean desencadenados por las incretinas.

Si bien los resultados analizados muestran que los análogos de la incretina, y específicamente la liraglutida, que es el análogo aprobado como coadyuvante para el manejo de la obesidad, son seguros y efectivos en un buen porcentaje de los pacientes tratados ya sean diabéticos o no, todavía deberá determinarse por qué la existencia de pacientes que no responden a la liraglutida como se ha observado en los grandes ensayos clínicos, así como también en un número pequeño de pacientes en la Clínica de Síndrome Metabólico, Prediabetes y DM2 de la Dirección de Investigaciones de la FOSCAL, donde unos individuos con sobrepeso y obesidad, especialmente aquellos con menores niveles de IMC responden positivamente a dosis entre 0,6 y $1,8 \mathrm{mg} /$ día de la liraglutida con pérdidas de peso que oscilan entre $2 \mathrm{y}$ $7 \mathrm{Kg}$, pérdida que se observa en las primeras semanas de tratamiento, además, de significativas disminuciones en los perímetros abdominal y de cuello, mientras otros, especialmente aquellos con IMC superiores a 30 , no tienen ninguna respuesta ni en el consumo de alimentos ni en la pérdida de peso, por lo que creemos necesario la realización de estudios locales que nos permitan evaluar la eficacia de la droga en nuestro medio, así como también evaluar el costo-efectividad de este nuevo tratamiento. Además, la aprobación de la FDA al uso de la liraglutida como droga antiobesidad es a dosis de $3 \mathrm{mg} /$ día por vía subcutánea, presentación que no existe en Colombia y que no ha sido aprobada por el INVIMA.

\section{Conclusiones}

La caracterización del eje entero-pancreático y la disponibilidad de análogos agonistas del GLP-1 han abierto interesantes alternativas terapéuticas para el manejo de la DM2 y del sobrepeso y la obesidad, asociados o no a alteraciones de la glucemia. No obstante, la pérdida de peso en los estudios realizados, especialmente con la liraglutida, es relativamente modesta por lo que su uso debería implementarse temprano en individuos con sobrepeso y obesidad abdominal y como coadyuvante a la implementación de programas de aumento en la actividad física, dieta saludable y soporte psico-social.

En conclusión, si bien parece que los incretino-miméticos pueden ser una alternativa farmacológica para el manejo del sobrepeso y la obesidad quedan todavía muchos interrogantes de su verdadera utilidad en nuestro medio.

\section{Responsabilidades éticas}

Protección de personas y animales. Los autores declaran que para esta investigación no se han realizado experimentos en seres humanos ni en animales.

Confidencialidad de los datos. Los autores declaran que en este artículo no aparecen datos de pacientes.

Derecho a la privacidad y consentimiento informado. Los autores declaran que en este artículo no aparecen datos de pacientes.

\section{Financiación}

José López-López y Karina A. Di Stefano reciben soporte del programa de Jóvenes Investigadores e Innovadores de Departamento Administrativo de Ciencia y TecnologíaCOLCIENCIAS-. 


\section{Conflicto de intereses}

El trabajo de J. López-López y K. Di Stefano es soportado financieramente por la Fundación Oftalmológica de Santander - FOSCAL, Universidad de Santander - UDES y por el Departamento Administrativo de Ciencia y Tecnología -COLCIENCIAS- a través del programa de formación de Jóvenes Investigadores e Innovadores.

\section{Bibliografía}

1. López-Jaramillo P, Lahera V, López-López J. Epidemic of cardiometabolic diseases: a Latin American point of view. Ther Adv Cardiovasc Dis. 2011;5:119-31.

2. Yusuf S, Hawken S, Ounpuu S, Dans T, Avezum A, Lanas F, et al. Effect of potentially modifiable risk factors associated with myocardial infarction in 52 countries (the INTERHEART study): case-control study. Lancet. 2004;364:937-52.

3. López-Jaramillo P, Sánchez RA, Díaz M, Cobos L, Bryce A, Parra Carrillo JZ, et al. Latin American consensus on hypertension in patients with diabetes type 2 and metabolic syndrome. J Hypertens. 2013;3:223-38.

4. O’Donnell MJ, Xavier D, Liu L, Zhang H, Chin SL, Rao-Melacini P, et al. Risk factors for ischaemic and intracerebral hemorrhagic stroke in 22 countries (the INTERSTROKE study): a case-control study. Lancet. 2010;376:112-23.

5. Rueda-Clausen CF, Silva FA, López-Jaramillo P. Epidemic of overweight and obesity in Latin America and the Caribbean. Int $\mathrm{J}$ Cardiol. 2008;125:111-2.

6. López-Jaramillo P, Herrera E, García RG, Camacho PA, CastiIlo VR. Inter-relationships between body mass index, C-reactive protein and blood pressure in a Hispanic pediatric population. Am J Hypertens. 2008;21:527-32.

7. García RG, Rincón MY, Arenas WD, Silva SY, Reyes LM, Ruiz SL, et al. Hyperinsulinemia is a predictor of new cardiovascular events in Colombian patients with a first myocardial infarction. Int J Cardiol. 2011;148:85-90.

8. López-Jaramillo P, Gómez-Arbeláez D, López-López J, LópezLópez C, Martínez-Ortega J, Gómez-Rodríguez A, et al. The role of leptin/adiponectin ratio in metabolic syndrome and diabetes. Horm Mol Biol Clin Investig. 2014;18:37-45.

9. Rueda-Clausen CF, Lahera V, Calderón J, Bolívar IC, Castillo VR, Gutiérrez M, et al. The presence of abdominal obesity is associated with changes in vascular function independently of other cardiovascular risk factors. Int J Cardiol. 2010;139: $32-41$.

10. International Diabetes Federation. IDF Diabetes Atlas, 6 th edn. Brussels, Belgium: International Diabetes Federation, 2013. http://www.idf.org/diabetesatlas.

11. King H, Aubert RE, Herman WH. Global burden of diabetes, 1995-2025: prevalence, numerical estimates, and projections. Diabetes Care. 1998;21:1414-31.

12. Hogan P, Dall T, Nikolov P. Economic costs of diabetes in the US in 2002. Diabetes Care. 2003;26:917-32.

13. López-Jaramillo P, Rey J, Gómez-Arbeláez D, Rodríguez Y, López-López J. Combatir la epidemia de diabetes mellitus tipo 2 en Latinoamérica: caracteristicas especiales que demandan acciones innovadoras. Clin Invest Arterioscl. 2011;23:90-9.

14. República de Colombia MPS. Encuesta Nacional de Salud. Hallado en: http://www.minsalud.gov.co/salud/Paginas/ EncuestaNacionaldeSaludPublica.aspx2010.[cited 2015. 14 de febrero].

15. Unger RH, Eisentraut AM. Entero-insular axis. Archives of internal medicine. 1969;123:261-6.

16. Baggio LL, Drucker DJ. Biology of incretins: GLP-1 and GIP. Gastroenterology. 2007;132:2131-57.
17. Meier JJ, Nauck MA. Incretins and the development of type 2 diabetes. Curr Diab Rep. 2006;6:194-201.

18. Nauck M, Stockmann F, Ebert R, Creutzfeldt W. Reduced incretin effect in type 2 (non-insulin-dependent) diabetes. Diabetología. 1986;29:46-52.

19. Rachman J, Barrow BA, Levy JC, Turner RC. Near-normalisation of diurnal glucose concentrations by continuous administration of glucagon-like peptide-1 (GLP-1) in subjects with NIDDM. Diabetología. 1997;40:205-11.

20. Bell GI, Santerre RF, Mullenbach GT. Hamster preproglucagon contains the sequence of glucagon and two related peptides. Nature. 1983;302:716-8. Epub 1983/04/21.

21. Orskov C, Wettergren A, Holst JJ. Biological effects and metabolic rates of glucagonlike peptide-1 7-36 amide and glucagonlike peptide-1 7-37 in healthy subjects are indistinguishable. Diabetes. 1993;42:658-61.

22. Knudsen LB, Nielsen PF, Huusfeldt PO, Johansen NL, Madsen K, Pedersen FZ, et al. Potent derivatives of glucagon-like peptide-1 with pharmacokinetic properties suitable for once daily administration. J Med Chem. 2000;43:1664-9.

23. Elbrond B, Jakobsen G, Larsen S, Agerso H, Jensen LB, Rolan P, et al. Pharmacokinetics, pharmacodynamics, safety, and tolerability of a single-dose of NN2211, a long-acting glucagon-like peptide 1 derivative, in healthy male subjects. Diabetes Care. 2002;25:1398-404.

24. Knudsen L, Knudsen S, Wilken M. Plasma protein binding of NN2211, a long-acting derivative of GLP-1, is important for its efficacy. Diabetes. 2003;52 Suppl 1:A321-2.

25. Malm-Erjefalt M, Bjornsdottir I, Vanggaard J, Helleberg H, Larsen $U$, Oosterhuis $B$, et al. Metabolism and excretion of the once-daily human glucagon-like peptide-1 analog liraglutide in healthy male subjects and its in vitro degradation by dipeptidyl peptidase IV and neutral endopeptidase. Drug Metab. Dispos. 2010;38:1944-53.

26. Jacobsen LV, Hindsberger C, Robson R, Zdravkovic M. Effect of renal impairment on the pharmacokinetics of the GLP-1 analogue liraglutide. Br J Clin Pharmacol. 2009;68:898-905.

27. Malm-Erjefalt M, Ekblom M, Brønsted L, Vouis J, Lennernas $\mathrm{H}$, Zdravkovic M. A randomised, double-blind, cross-over trial investigating the effect of liraglutide on the absorption pharmacokinetics of concomitantly administered oral drugs in healthy subjects. Diabetes. 2008;57 Suppl 1:A130.

28. Holz GHt, Kuhtreiber WM, Habener JF. Induction of glucose competence in pancreatic beta cells by glucagon-like peptide1(7-37). T Assoc Am Physician. 1992;105:260-7.

29. Bode HP, Moormann B, Dabew R, Goke B. Glucagon-like peptide 1 elevates cytosolic calcium in pancreatic beta-cells independently of protein kinase A. Endocrinology. 1999;140:3919-27.

30. MacDonald PE, Salpatek AM, Wheeler MB. Glucagon-Like Peptide-1 Receptor Activation Antagonizes Voltage-Dependent Repolarizing K Currents in -Cells. Diabetes. 2002;51 Suppl 3:S443-7.

31. Drucker DJ, Philippe J, Mojsov S, Chick WL, Habener JF. Glucagon-like peptide I stimulates insulin gene expression and increases cyclic AMP levels in a rat islet cell line. P Natl Acad Sci Usa. 1987;84:3434-8.

32. Koob GF. Drugs of abuse: anatomy, pharmacology and function of reward pathways. Trends Pharmacol Sci. 1992;13:177-84.

33. Wise RA. Role of brain dopamine in food reward and reinforcement. Philos T Roy Soc B. 2006;361:1149-58.

34. Wise RA. Dopamine and food reward: back to the elements. Am J Physiol-Reg I. 2004;286:R13.

35. Larsen PJ, Tang-Christensen M, Holst JJ, Orskov C. Distribution of glucagon-like peptide-1 and other preproglucagon-derived peptides in the rat hypothalamus and brainstem. Neuroscience. 1997;77:257-70.

36. Grill HJ, Hayes MR. The nucleus tractus solitarius: a portal for visceral afferent signal processing, energy status assessment 
and integration of their combined effects on food intake. Int J Obes (Lond). 2009;33 Suppl 1:S11-5.

37. Shughrue PJ, Lane MV, Merchenthaler I. Glucagon-like peptide-1 receptor (GLP1-R) mRNA in the rat hypothalamus. Endocrinology. 1996;137:5159-62.

38. Hayes MR, Bradley L, Grill HJ. Endogenous hindbrain glucagonlike peptide-1 receptor activation contributes to the control of food intake by mediating gastric satiation signaling. Endocrinology. 2009;150:2654-9.

39. Nauck MA, Niedereichholz U, Ettler R, Holst JJ, Orskov C, Ritzel $\mathrm{R}$, et al. Glucagon-like peptide 1 inhibition of gastric emptying outweighs its insulinotropic effects in healthy humans. Am J Physiol. 1997;273:E981-8.

40. Drucker DJ, Nauck MA. The incretin system: glucagon-like peptide- 1 receptor agonists and dipeptidyl peptidase- 4 inhibitors in type 2 diabetes. Lancet. 2006;368:1696-705.

41. Vilsboll T, Zdravkovic M, Le-Thi T, Krarup T, Schmitz O, Courreges JP, et al. Liraglutide, a long-acting human glucagon-like peptide-1 analog, given as monotherapy significantly improves glycemic control and lowers body weight without risk of hypoglycemia in patients with type 2 diabetes. Diabetes Care. 2007;30:1608-10.

42. Marre M, Shaw J, Brandle M, Bebakar WM, Kamaruddin NA, Strand J, et al. Liraglutide, a once-daily human GLP-1 analogue, added to a sulphonylurea over 26 weeks produces greater improvements in glycaemic and weight control compared with adding rosiglitazone or placebo in subjects with Type 2 diabetes (LEAD-1 SU). Diabetic Med. 2009;26:268-78.

43. Hansen BC, Bjenning C, Bjerre Knudsen L. Sustained appetite suppression and weight loss in obese rhesus monkeys treated with a long-acting GLP-1 derivative, NN2211. Diabetologia. 2001;44:A196.

44. Guo N, Sun J, Chen H, Zhang H, Zhang Z, Cai D. Liraglutide prevents diabetes progression in prediabetic OLETF rats. Endocr J. 2013;60:15-28.

45. Cummings BP, Stanhope KL, Graham JL, Baskin DG, Griffen SC, Nilsson C, et al. Chronic administration of the glucagon-like peptide- 1 analog, liraglutide, delays the onset of diabetes and lowers triglycerides in UCD-T2DM rats. Diabetes. 2010;59:2653-61.

46. Inoue K, Maeda N, Kashine S, Fujishima Y, Kozawa J, HiugeShimizu A, et al. Short-term effects of liraglutide on visceral fat adiposity, appetite, and food preference: a pilot study of obese Japanese patients with type 2 diabetes. Cardiovasc Diabetol. 2011;10:109.

47. Nauck M, Frid A, Hermansen K, Shah NS, Tankova T, Mitha IH, et al. Efficacy and safety comparison of liraglutide, glimepiride, and placebo, all in combination with metformin, in type 2 diabetes: the LEAD (liraglutide effect and action in diabetes)-2 study. Diabetes Care. 2009;32:84-90.

48. Rosenstock J, Klaff LJ, Schwartz S, Northrup J, Holcombe JH, Wilhelm K, et al. Effects of exenatide and lifestyle modification on body weight and glucose tolerance in obese subjects with and without pre-diabetes. Diabetes Care. 2010;33:1173-5.

49. Mari A, Degn K, Brock B, Rungby J, Ferrannini E, Schmitz O. Effects of the long-acting human glucagon-like peptide-1 analog liraglutide on beta-cell function in normal living conditions. Diabetes Care. 2007;30:2032-3.

50. Matthews D, Marre M, Le-Thi T, Zdravkovic M, Simo R, Garber A, et al. Liraglutide, a human GLP-1 analogue, significantly improves beta cell function in subjects with type 2 diabetes. Diabetologia. 2008;51(Supp 1):S892.

51. Pucci A, Finer N. New Medications for Treatment of Obesity: Metabolic and Cardiovascular Effects. Can J Cardiol. 2015;31:142-52.
52. Pi-Sunyer X, Astrup A, Fujioka K, Greenway F, Halpern A, Krempf $\mathrm{M}$, et al. A randomized, controlled trial of $3.0 \mathrm{mg}$ of liraglutide in weight management. N Engl J Med. 2015;373:11-22.

53. Davies MJ, Bergenstal R, Bode B, Kushner RF, Lewin A, Vang Skjoth T, et al. Efficacy of liraglutide for weight loss among patients with type 2 diabetes. The SCALE diabetes randomized clinical trial. JAMA. 2015;314: 687-99.

54. Wadden TA, Hollander P, Klein S, Niswender K, Woo V, Hale $P M$, et al. Weigh maintenance and additional weigh loss with liraglutide after low-calorie-diet-induce weigh loos: The SCALE maintenance randomized study. Int J Obes (Lond). 2013;37:1443-51.

55. Garber A, Henry R, Ratner R, García-Hernández PA, RodríguezPattzi H, Olvera-Álvarez I, et al. Liraglutide versus glimepiride monotherapy for type 2 diabetes (LEAD-3 Mono): a randomised, 52-week, phase III, double-blind, parallel-treatment trial. Lancet. 2009;373:473-81.

56. Barnett AH. The role of GLP-1 mimetics and basal insulin analogues in type 2 diabetes mellitus: guidance from studies of liraglutide. Diabetes Obes Metab. 2012;14: 304-14.

57. Rigato M, Fadini GP. Comparative effectiveness of liraglutide in the treatment of type 2 diabetes. Diabetes Metab Syndr Obes. 2014;7:107-20.

58. Zinman B, Gerich J, Buse JB, Lewin A, Schwartz S, Raskin P, et al. Efficacy and safety of the human glucagon-like peptide-1 analog liraglutide in combination with metformin and thiazolidinedione in patients with type 2 diabetes (LEAD-4 Met + TZD). Diabetes Care. 2009;32:224-30.

59. Russell-Jones D, Vaag A, Schmitz O, Sethi BK, Lalic N, Antic $\mathrm{S}$, et al. Liraglutide vs. insulin glargine and placebo in combination with metformin and sulfonylurea therapy in type 2 diabetes mellitus (LEAD- 5 met + SU): a randomised controlled trial. Diabetología. 2009;52:2046-55.

60. Fonseca VA, Devries JH, Henry RR, Donsmark M, Thomsen HF, Plutzky J. Reductions in systolic blood pressure with liraglutide in patients with type 2 diabetes: insights from a patient-level pooled analysis of six randomized clinical trials. J Diabetes Complications. 2014;28:399-405.

61. Robinson LE, Holt TA, Rees K, Randeva HS, O’Hare JP. Effects of exenatide and liraglutide on heart rate, blood pressure and body weight: systematic review and meta-analysis. BMJ Open. 2013;3(1.).

62. Gutzwiller JP, Tschopp S, Bock A, Zehnder CE, Huber AR, Kreyenbuehl $M$, et al. Glucagon-like peptide 1 induces natriuresis in healthy subjects and in insulin resistant obese men. J Clin Endocr Metab. 2004;89:3055-61.

63. Kim M, Platt MJ, Shibasaki T, Quaggin SE, Backx PH, Seino S, et al. GLP-1 receptor activation and Epac2 link atrial natriuretic peptide secretion to control of blood pressure. Nat Med. 2013;19:567-75.

64. Marso SP, Poulter NR, Nissen SE, Nauck MA, Zinman B, Daniels $\mathrm{GH}$, et al. Design of the liraglutide effect and action in diabetes: evaluation of cardiovascular outcome results (LEADER) trial. Am Heart J. 2013;166:823-30.

65. Vilsboll T, Christensen M, Junker AE, Knop FK, Gluud LL. Effects of glucagon-like peptide-1 receptor agonists on weight loss: systematic review and meta-analyses of randomised controlled trials. BMJ. 2012;344:d7771.

66. Astrup A, Rossner S, Van Gaal L, Rissanen A, Niskanen L, Al Hakim M, et al. Effects of liraglutide in the treatment of obesity: a randomised, double-blind, placebo-controlled study. Lancet. 2009;374:1606-16.

67. Astrup A, Carraro R, Finer N, Harper A, Kunesova M, Lean ME, et al. Safety, tolerability and sustained weight loss over 2 years 
with the once-daily human GLP-1 analog, liraglutide. Int J Obes (Lond). 2012;36:843-54.

68. Parks M, Rosebraugh C. Weighing Risks and Benefits of Liraglutide - The FDA's Review of a New Antidiabetic Therapy. N Engl J Med. 2010:774-7.

69. Egan AG, Blind E, Dunder K, Graeff PA, Hummer T, Bourcier T, et al. Pancreatic Safety of incretin-based drugs, FDA and EMA assessment. N Engl J Med. 2014;370(9):794-7.

70. Raz I, Bhatt DL, Hirshberg B, Mosenzon O, Scirica BM, UmezEronini A, et al. Incidence of pancreatitis and pancreatic cancer in a randomized controlled multicenter trial (SAVOR-TIMI 53) of the dipeptidyl peptidase-4 inhibitor saxagliptin. Diabetes Care. 2014;37:2435-41.
71. White WB, Cannon CP, Heller SR, Nissen SE, Bergenstal RM, Bakris GL, et al. Alogliptinafter acute coronary syndrome in patients with type 2 diabetes. N Engl J Med. 2013;369: 1327-35.

72. Alves C, Batel-Marques F, Macedo AF. A meta-analysis of serious adverse events reported with exenatide and liraglutide: Acute pancreatitis and cancer. Diabetes Reser Clin Pract. 2012;98:271-84. 